University of Wollongong

Research Online

Faculty of Commerce - Papers (Archive)

Faculty of Business and Law

$1-6-2008$

\title{
The low stability of brand-attribute associations is partly due to market research methodology
}

Sara Dolnicar

University of Wollongong, s.dolnicar@uq.edu.au

J. R. Rossiter

University of Wollongong, jrossite@uow.edu.au

Follow this and additional works at: https://ro.uow.edu.au/commpapers

Part of the Business Commons, and the Social and Behavioral Sciences Commons

\section{Recommended Citation}

Dolnicar, Sara and Rossiter, J. R.: The low stability of brand-attribute associations is partly due to market research methodology 2008.

https://ro.uow.edu.au/commpapers/464

Research Online is the open access institutional repository for the University of Wollongong. For further information contact the UOW Library: research-pubs@uow.edu.au 


\title{
The low stability of brand-attribute associations is partly due to market research methodology
}

\author{
Abstract \\ Using an experiment built into a longitudinal survey, we demonstrate that the low stability of consumers' \\ brand-attribute associations (see Rungie et al., 2005) can be partly explained by the prevailing methods \\ used in market research, which can often lead consumers to construct temporary associations. To \\ increase the proportion of stable brand-attribute associations, we recommend the following \\ improvements in market research methods: use of a shorter, brand-prompted attribute association task; \\ inclusion of an "don't know this brand" option to isolate ratings of brands unknown to the individual; \\ omission of the standard instruction to guess when uncertain; and, in cross-cultural studies, translation of \\ instructions and attribute descriptions into appropriate first languages. Even with these improvements, \\ however, the maximal stability of associations that brands can achieve is less than $100 \%$ after correcting \\ for methodological influences. This imperfect stability may mean that consumers learn brand-specific \\ attribute associations that are temporary but stable enough to lead them to try or re-try the brand, after \\ which consumers replace the specific brand associations with a summary brand attitude.

\section{Disciplines} \\ Business | Social and Behavioral Sciences

\section{Publication Details} \\ This article was originally published as Dolnicar, S, Rossiter, JR, The low stability of brand-attribute \\ associations is partly due to market research methodology. International Journal of Research in \\ Marketing, 25 (2), 2008, 104-108.
}




\title{
The low stability of brand-attribute associations
}

\section{is partly due to market research methodology}

\author{
Sara Dolnicar $^{* 1}$, John R. Rossiter \\ Marketing Research Innovation Centre, Faculty of Commerce \\ University of Wollongong, Northfields Avenue, Wollongong, Australia
}

\section{Acknowledgements}

This study was funded primarily by the Australian Research Council (ARC) and by the University of Wollongong under the New Partnership Grant Scheme. We thank Martin O'Shannessy, former CEO of the IRIS research company and now CEO of the Newspoll corporation, for additional financial support. We also thank Maria Heindler of the University of Vienna, and Stewart Rossiter and Edmund Goh of the University of Wollongong for their help as research assistants for this project. Special thanks also to IJRM Co-Editor Donald R. Lehmann for helpfully suggesting how to condense this report and to an anonymous IJRM technical editor.

\footnotetext{
Abstract

* Authors listed in alphabetical order.

${ }^{1}$ Corresponding author. Sara Dolnicar, Tel: ++61 (2) 4221 3862, Fax: ++61 (2) 4221 4154, email: sara_dolnicar@uow.edu.au.
} 
Using an experiment built into a longitudinal survey, we demonstrate that the low stability of consumers' brand-attribute associations (see Rungie et al., 2005) can be partly explained by the prevailing methods used in market research, which can often lead consumers to construct temporary associations. To increase the proportion of stable brand-attribute associations, we recommend the following improvements in market research methods: use of a shorter, brand-prompted attribute association task; inclusion of an "don't know this brand" option to isolate ratings of brands unknown to the individual; omission of the standard instruction to guess when uncertain; and, in cross-cultural studies, translation of instructions and attribute descriptions into appropriate first languages. Even with these improvements, however, the maximal stability of associations that brands can achieve is less than $100 \%$ after correcting for methodological influences. This imperfect stability may mean that consumers learn brand-specific attribute associations that are temporary but stable enough to lead them to try or re-try the brand, after which consumers replace the specific brand associations with a summary brand attitude. 


\section{Introduction}

Three years ago, Rungie, Laurent, Dall'Olmo Riley, Morrison, and Roy (2005) published in IJRM a meta-analysis conducted on eight data sets taken from studies by Ehrenberg and colleagues. The main finding in all eight data sets was that the brand-attribute associations that consumers report in the "free choice" affirmative binary rating tasks ("yes" only, scored 1 or 0 ), which are typical of commercial market research surveys, show remarkably low stability. Between two interviews with the same consumers, spaced an average of three months apart, the average association chosen on one interview was chosen on the other - that is, chosen stably only $13 \%$ of the time. We inferred this percentage from Table 4 (p. 316) of Rungie et al., taking into account their observed average initial response rate of approximately $25 \%$.

We have taken a slightly different approach to analyze the stability of brandattribute associations. The analysis of Rungie et al. produces stability estimates that are too low because they use as their base all possible associations, including the many "00s" (double zeroes) in the data, which are not associations at all and have the effect of severely deflating the percentage of "11s" (double positives). Our measure of stability takes associations made as the base, such that stability $=11 /(01+10+11) \mathrm{x}$ $100 \%$, where ' 11 ' refers to the frequency of associations made in the first and second interviews, and ' 01 ' indicates, for example, the frequency of associations made in the first interview but not in the second. In addition, our formula takes the average of the "forward" repeat rate and the "backward" repeat rate, since instability can occur forwards (10) or backwards (01) during the two interviews. In contrast, the analysis of Rungie et al. uses only the forward repeat rate, which gives a "one-way" average 
stability of $49 \%$ over an average interval of approximately 12 weeks (Rungie et al., p. $311)$.

In our study, consecutive interviews were held one week apart to increase the likelihood that responses would be stable. The results of our study produced an average "two-way" stability estimate of 53\%. Although slightly higher than the value obtained by Rungie et al., this estimate means that nearly half (47\%) of brandattribute associations were unstable; in other words, they were made in the first survey but not in the second one conducted a week later, or they were made only on the second occasion. In other words, only about half of the content of the average brand's image is stably held by the average consumer and is therefore valid. We suspect that many of the temporary associations making up the rest of the brand's image, are induced by the research method.

In the present study, we investigate the aspects of market research methods that are most likely to affect the observed stability of brand-attribute associations. We conduct an experiment within the context of a longitudinal survey involving the same respondents. Our results identify several factors of research methodology that may be partly responsible for the low stability of associations, and some of these were also pointed out by Rungie and colleagues (2005).

\section{Factors hypothesized to affect brand-attribute stability}

Brand-image survey researchers assume that respondents are retrieving established associations from memory. But a likely reason for observing unstable brand-attribute associations is that many survey respondents construct them "on the spot" because they feel they "should know" the attributes of most brands they have heard of. Krosnick, Narayan, and Smith (1996) found that temporary constructions 
arise frequently in public opinion surveys, so they may also be a problem in market research surveys. Payne, Bettman, and Johnson (1992) found that consumers are willing to construct overall brand preferences for brands they know little about, and it seems likely that consumers in surveys also manufacture brand-attribute associations. In the present study, we investigated six methodological factors intended to discourage temporary construction of brand-attribute associations and therefore lead to higher stability estimates. We manipulated two likely methodological factors task ease and direction of association - and measured four others. These six factors were chosen as the basis for the hypotheses in our study for the reasons described below.

\subsection{Task ease}

In the typical brand-image survey, as in the data sets analyzed by Rungie et al. (2005), consumers are presented with eight or so brands and 12 or so attribute statements, meaning that they have to make about 100 judgments. A task as long as this is likely to induce fatigue, and fatigue causes more "random" responding and thus temporary associations. Data quality has long been known to suffer in proportion to respondent fatigue (Johnson, Lehmann, \& Horne, 1990). Fatigue seems especially likely to be a factor when respondents cannot easily terminate the task, as when they are members of a paid panel (Rathod \& LaBruna, 2005; and the data sets in Rungie et al., 2005) or, in our case, are "captive" students. In such circumstances, the respondent's response level (the number of associations chosen) is likely to stay up while the overall stability of these associations drops because of temporary constructions that are less carefully considered. A shorter and thus easier task should increase observed stability. 
H1. Task ease (relatively few brands and attributes) will increase the incidence of stable associations.

\subsection{Consumer involvement}

A second factor that might affect stability is the surveyed consumer's interest in, or involvement with, the product category. Rungie et al. (2005) also suggested the importance of this factor. Less involved consumers, especially if they are not the usual buyer or principal user of the product, may report associations that are less valid, and therefore less stable, than those reported by more involved consumers.

H2. Consumer involvement with the product category will increase the incidence of stable associations.

\subsection{Brand familiarity}

A third factor that may affect stability, also suggested by Rungie et al. (2005), is when consumers are asked to rate brands with which they have little familiarity. The brand name itself may seem familiar to them and so they may feel justified in assigning attributes to the brand that they infer from other brands that they know in the product category. But the low actual familiarity would seem to encourage guessing; indeed, in most commercial market research surveys, which employ the “free choice” method, respondents are instructed: “... if you don't know, please guess" (Joyce, 1963, p. 60). Guessed associations, being temporary constructions, are likely to be unstable.

H3. Greater consumer familiarity with the brand will increase the incidence of stable associations.

\subsection{Attribute importance}


A fourth factor that may affect stability, which Rungie et al. also mentioned, is the perceived importance of the attributes that can be associated with the brands. In principle, attribute importance should not affect whether the respondent chooses the attribute on either occasion, because an association is an association no matter how trivial the attribute (Carpenter, Glazer, \& Nakamoto, 1994). However, attribute importance may "focus" the respondent to choose with greater consistency.

H4. Higher perceived importance of an attribute will increase how often it is stably associated with the brand.

\subsection{Association direction}

A fifth factor that may affect the stability of brand-attribute associations is the direction of the association. Invariably in commercial market research surveys, such as those analyzed by Rungie et al. (2005), associations are attribute-prompted in that an attribute is presented first and then the respondent is asked to indicate which of a list of brands "has" that attribute (McDonald, 2000). However, brand images are widely thought to be stored holistically in memory (Dillon, Madden, Kirmani, \& Mukherjee, 2001; Keller, 2003), and therefore their attribute associations should be retrieved more readily if a brand prompt is presented first and the list of attributes afterward.

H5. Brand-prompted associations will increase the incidence of stable associations.

\subsection{English comprehension}

Attributes in brand-image surveys are typically stated in one to five words and in English, as in the British and U.S. studies analyzed by Rungie et al. (2005). 
English, however, is not the native language of a large and growing proportion of consumers in either of these countries. Less than perfect comprehension of English may be a sixth factor that lowers stability because non-native speakers' interpretation of the attribute description may be inconsistent. In support of this contention, Carstairs, Myors, Shore, and Fogarty (2006) found that adult respondents for whom English was a second language performed significantly worse than native English speakers on English-language tests of cognitive abilities, even after controlling for education level.

H6. High English comprehension ability will increase the incidence of stable associations.

\section{Method}

We designed a brand-image survey and administered it to the same consumers on two occasions a week apart. (In reality, the survey was administered three times, but we report here only the stability over the first two occasions, in keeping with the practice of previous studies.) We experimentally manipulated two hypothesized factors - task ease and association direction - and measured the four other hypothesized factors as individual-difference variables.

\subsection{Participants}

Participants in the study were undergraduate business students at an Australian university. The final sample of participants for whom complete data on all measured variables was obtained was $\mathrm{N}=357$.

\subsection{Products, brands, and attributes}


Because the participants were students, we selected two products with which they were likely to have some familiarity - sports shoes and laundry detergents - but in which interest or involvement was likely to vary between individuals. These two products represent the categories of durables and fast-moving consumer goods, which were the categories covered in the meta-analysis of Rungie et al. (2005).

Brands and attributes in both product categories were selected based on openended interviews with separate samples of about 50 students. For each category, five relatively "strong-image" brands and five "weak-image" brands were chosen from the students' nominations. For each category, after merging obviously redundant references to attributes, the eight most frequently mentioned attributes and four low frequency attributes were chosen such that the attributes in the study would vary widely in importance to the students. The brands and attribute descriptions are given in Table 1.

Table 1 about here

\subsection{Questionnaire versions}

Randomly on the first occasion, but then consistently thereafter, each participant received either a short questionnaire (one product, five brands and six attributes) or a long questionnaire (both products, 10 brands and 12 attributes for each). Brands and attributes were fully rotated across respondents in both questionnaire versions. In addition, approximately half the questionnaires were worded with attribute prompting in the association task and the other half with brand prompting, and this was held constant for each respondent. 


\subsection{Measures}

The dependent variable, brand-attribute association stability, was measured as the number of brand-attribute associations made by the respondent on both surveys (11) expressed as a percentage of the total number of associations made on either survey $(01+10+11)$. This is the proportion of "double positive" associations among all actual associations.

The six hypothesized methodological factors, i.e. the independent variables, were measured as follows. The surveys manipulated Task ease as follows. The short questionnaire included only five brands randomly selected from the original 10, and six attributes (four high-frequency and two low-frequency), thereby requiring only 30 judgments. In contrast, the long questionnaire included 10 brands and 12 attributes, and thereby required 120 judgments, which is approximately the upper limit number in the data sets analyzed by Rungie et al. A manipulation check item asking "how tiring" the questionnaire was produced mean ratings of 7.8 for the short questionnaire and 11.4 for the long questionnaire using a scale of $1=$ "not at all tiring" to $20=$ "very tiring" ( $p<.0001)$. Consumer involvement was measured by having each respondent rate his or her degree of interest in the product category along five characteristics taken from the involvement scale of Zaichkowsky (1985), scored from $0=$ "low" to $20=$ "high," and summed to yield a score range of 0 to 100. Brand familiarity was measured for individual respondents and brands on a verbal scale scored as $1=$ "never heard of it," 2 = "heard of it but don't know much about it," $3=$ "quite familiar," and 4 = "very familiar." In addition, the respondent was asked to nominate his or her "favorite" brand in the product categories of sports shoes and laundry detergents, depending on which product category was on the questionnaire. Attribute importance was rated by each respondent on a scale from $0=$ "low 
importance" to $20=$ "high importance." Association direction was either attributeprompted (attribute first, brands listed) or brand-prompted (brand first, attributes listed). This was achieved in the two questionnaire versions by presenting, in the first case, each attribute above a row of brands and, in the second case, each brand above a row of attributes. Respondents were instructed to answer along the rows, putting a tick (check) in the box to signify a perceived association and leaving the answer box blank otherwise. English comprehension was proxied by asking respondents to indicate whether English (the language of the questionnaire) was their first language or not. Our sample included $40 \%$ for whom English was not their first language.

\section{Results}

We present our results showing the effects of the methodological factors on brand-attribute association stability first from a simple univariate perspective, which does not allow for possible interactions of the factors (e.g., interaction of English comprehension difficulty with task length), and then as a multivariate combination of the factors.

For all analyses, the number of observations was 8,320 . This represents the number of respondents $(\mathrm{N}=357)$ times the average number of associations they made at least once (23).

\subsection{Univariate results}

To render the univariate results easier to interpret, we made all the methodological factors binary by dichotomizing the three continuous variables: for brand familiarity, low = never heard of it, or heard of it but know little about it, and high $=$ quite or very familiar; for attribute importance, low $=0$ to 10 , high $=11$ to 20 ; 
and for consumer involvement, a median split of low $=0$ to 45 , high $=46$ to 100 . The results, including the results from the supplementary brand familiarity measure of "favorite" brand, are shown in Table 2. When interpreting the results, it is helpful to keep in mind that the observed mean "double positive" level of stability for our entire data set was $53 \%$.

Table 2 about here

The differences in stability are large for the brand familiarity factor, particularly for the most extreme factor of favorite vs. non-favorite brand, as well as for task ease, English comprehension, and association direction. Because the observations, totalling 8,320, are not independent, we adopted a conservative significance level of $.05 / 25$, or $p<.002$, which reflects the base of 357 independent respondents. The two methodological factors that gave results contrary to our hypotheses are consumer involvement with the product, and attribute importance, which are not associated with significant differences in stability at the conservative significance level of $p<.002$.

Interestingly, the stability of brand-attribute associations varies by category and type. When we classified attributes as performance vs. image, stability of performance attributes averaged 52\% for both laundry detergents and sport shoes. However, for image attributes, stability remained about the same $(50 \%)$ for the product of greater interest to the students (sport shoes), whereas it dropped to $29 \%$ for the product of less interest (laundry detergents). This result implies, unsurprisingly, 
that the stability of image attributes will be low for low-involvement, utilitarian product categories.

\subsection{Multivariate results}

The seven methodological factors (seven including favorite brand) were entered into a binary logistic regression model to assess their combined significance as predictors of stable associations. Double positive stability was the binary dependent variable: $1=$ stable, $0=$ unstable. For this analysis, the three continuous predictor variables were used, not their binary forms, while the other four predictor variables were inherently binary. This produced a regression model showing good fit to our data set according to the Hosmer and Lemeshow test, which is appropriate for binary logistic regression $\left(\chi^{2}=6.96, d f=8, p=.542\right)$. Also, no multicollinearity among the methodological factors was evident: correlations among the predictors were .5 or less. The results are given in Table 3 .

Table 3 about here

The multivariate results differ from the univariate results only in that consumer involvement with the product category becomes statistically significant. It is worth noting that the brand familiarity hypothesis was tested twice due to the inclusion of favorite brand on the questionnaires. As a result of the multivariate analysis, five of the six hypotheses are strongly supported, whereas the hypothesis about attribute importance is rejected. 
Next, we estimated average stability after controlling for the major methodological factors other than "favorite brand," which cannot be controlled for. To do this, we regressed the binary dependent variable of double positive associations on the dichotomously coded main predictor variables of task ease, brand familiarity, and English comprehension. This regression equation produced a maximum average stability estimate across all brands and attributes of $66 \%$ (taking all three predictors at their high dichotomous values) and a minimum of 32\% (taking all three at their low values).

Finally, we examined the peak stabilities for single brand-attribute associations in our data. We did this for each product under the conditions of the ideal cell, where the three factors of task ease, brand familiarity, and English comprehension have high values. Five of the peak stabilities reached $100 \%$ but they involved only a few respondents. Other very high stabilities were for associations that were easy to guess from the brand name (e.g., Home Brand - "Low-priced") or common knowledge (e.g., Nike - "American"). The peak estimates for associations that were actually targeted in the brand's advertising were for the Australian market leaders: for detergents, Omo - "Gets out tough stains," $81 \%$; and for sports shoes, Nike - "Comfortable," $82 \%$. These peak association stabilities were considerably less than $100 \%$, even though the interval between surveys was only one week.

\section{Discussion}

Our study has demonstrated that the low stability of consumers' brandattribute associations can be partly explained by aspects of market research methodology. Over the short interval of one week, we found that only $53 \%$ of the associations made on either survey were made twice, i.e. proved to be stable. This is 
only slightly higher than the $49 \%$ average stability (for the "forward" repeat rate) observed by Rungie et al. (2005) over longer intervals, during which time marketing communications for brands presumably changed and thereby caused consumers to form new associations and forget previous associations. The fact that stability was not much higher over the shorter interval in our study means that much of the instability is due to factors in the market research methodology.

When controllable methodological factors were applied, we found that the stability of actual associations as measured by our formula increased to $66 \%$ for the average association made over all brands and attributes. To approach this level of a two-thirds stability of brand-attribute associations, we recommend:

- Confining the survey to users of the product category (unless, of course, the surveyed brands are introducing a new category).

- Making the questionnaire as short as possible (only main brands and their advertised attributes).

- Inclusion of a "don't know this brand" option in addition to the affirmative binary ("yes" only) answer option so that ratings of unfamiliar brands, which are likely to be unstable, can be distinguished in the analysis.

- Omitting the standard instruction to guess when respondents do not know the association.

- Asking for brand-prompted attribute associations rather than the usual method of asking which brands are associated with one attribute, then another, and so on.

- In multi-cultural brand-image surveys, translating the instructions and attribute descriptions into respondents' first languages. 
Two other methodological factors are worth mentioning. One is that market research practitioners measuring brand images should use a continuous panel of consumers, rather than following the standard practice made popular by the world's leading advertising research company, Millward Brown, of interviewing a new sample of consumers each week. Unless the same respondents are tracked, stable associations cannot be distinguished from unstable associations. Our findings, and those of Rungie et al., suggest that as many as half of the associations recorded in commercial surveys are unstable, and these can be identified only by using a panel method.

The other methodological factor that is an important topic for future research is answer formats. All commercial surveys use the affirmative binary free-choice answer format ("yes" only), but it is worth testing a trinary forced-choice answer format ("yes," “no," “don't know”), because this would remove the ambiguity of whether lack of endorsement means “don't know" or alternatively represents the respondent's belief that the brand does not have the attribute. To draw an example from our questionnaire, a "no" option would allow consumers to tell the researcher that Dunlop sports shoes are not "cool." The Dunlop brand was reported by students to be "uncool" at the time of our pilot study. However, since then Dunlop's "Volley" shoes seem to have become "cool," a dramatic change that the trinary answer format could detect. At the other extreme are polytomous answer scales (multiple-category, typically 5- or 7-point), which are also forced-choice. These longer answer formats are often used by academic researchers to measure brand-attribute associations (beliefs) but are avoided by practitioners because respondents have to consider five or seven possible answers for each association, which practitioners expect would considerably increase the number of judgments and time taken for the survey. 
However, the research question remains of whether longer multiple-category answer scales would register associations more sensitively and therefore more stably. Rungie et al. do not expect that they will (2005, p. 317), but this needs to be tested empirically.

Even under ideal measurement conditions, peak stabilities are unlikely to reach $100 \%$. We observed peak stabilities for advertising-targeted associations of about $80 \%$ and Rungie et al. observed peak stabilities in some product categories that were much lower than this. Such findings undermine the assumptions of brand-image theorists, notably those of Keller (2003) in his influential textbook, but also those of most brand managers, who hold that brand-attribute associations can be established relatively permanently and have an ongoing causal influence on brand choice. We also found that performance attributes are more stably associated with lowinvolvement, utilitarian products, represented here by laundry detergents, than are image attributes. Nevertheless, not even these performance attribute associations are held stably by all consumers.

Our study reveals that the detection of valid brand-attribute associations is made difficult by market research methods that seem to encourage many false reports in the form of temporary constructions that then turn out to be unstable over a period as short as one week. Our recommended improvements in research methods can reduce the incidence of false reports while hopefully leaving intact valid but transient reports. These valid but transient reports can be distinguished from valid and stable reports by applying our "double positive" formula to panel-derived data.

In conclusion, we suggest a likely theoretical explanation of why valid brandattribute associations may be transient. The explanation may reside in the model of Howard (1977), called routinized response behavior (see also Lynch, Marmorstein, \& 
Weigold, 1988; Mazursky, 1990; and Rossiter, 1987). It may be that most consumers forget the attribute-based reasons why they chose or rejected the many brands they have considered and instead retain just a summary attitude sufficient to guide choice the next time.

\section{References}

Carpenter, G.S., Glazer, R., \& Nakamoto, K. (1994). Meaningful brands from meaningless differentiation. Journal of Marketing Research, 31(3), 339-350.

Carstairs, J.R., Myors, B., Shores, E.A., \& Fogarty, G. (2006). Influence of language background on tests of cognitive abilities: Australian data. Australian Psychologist, 41(1), 48-54.

Dillon, William R., Thomas J. Madden, Amna Kirmani, \& Soumen Mukherjee (2001). Understanding what's in a brand rating: a model for assessing brand and attribute effects and their relationship to brand equity. Journal of Marketing Research, 38(4), 415-429.

Howard, J.A. (1977). Consumer behavior: application of theory. New York: McGraw-Hill.

Johnson, M.D., Lehmann, D.R., \& Horne, D.R. (1990). The effects of fatigue on judgments of interproduct similarity. International Journal of Research in Marketing, 7(1), 35-43.

Joyce, T. (1963). Techniques of brand image measurement. In New developments in research (pp. 45-63). London: Market Research Society.

Keller, K.L. (2003). Strategic brand management. $2^{\text {nd }}$ edn. Upper Saddle River, NJ: Prentice Hall. 
Krosnick, J.A., Narayan, S., \& Smith, W.R. (1996). Satisficing in surveys: initial evidence. New Directions for Evaluation, 70(Summer), 29-44.

Lynch, J.G., Jr., Marmorstein, H., \& Weigold, M.F. (1988). Choices from sets including remembered brands: use of recalled attributes and prior overall evaluations. Journal of Consumer Research, 15(2), 169-184.

Mazursky, D. (1990). Temporal instability in the salience of behavioral intention predictors. Journal of Economic Psychology, 11(3), 383-402.

McDonald, C. (2000). Tracking advertising and monitoring brands. Admap monograph no. 6. Henley-on-Thames, UK: Admap Publications.

Payne, J.W., Bettman, J.R., \& Johnson, E.J. (1992). Measuring constructed preferences: a constructive processing approach. Annual Review of Psychology, 43, 87-131.

Rathod, S., \& LaBruna, A. (2005). Questionnaire length and fatigue effects. ESOMAR Panel Research Conference, Budapest, April. Electronic document available from ESOMAR, The Netherlands.

Rossiter, J.R. (1987). Comments on 'Consumer beliefs and brand usage' and on Ehrenberg's ATR model. Journal of the Market Research Society, 29(1), 8388.

Rungie, C., Laurent, G., Dall'Olmo Riley, F., Morrison, D.G., \& Roy, T. (2005). Measuring and modeling the (limited) reliability of free choice attitude questions. International Journal of Research in Marketing, 22(3), 309-318.

Zaichkowsky, J.L. (1985). Measuring the involvement construct. Journal of Consumer Research, 12(3), 341-352. 
Table 1

Brands and attributes for the two product categories used in the study. Highfrequency attributes are superscripted "a" and low-frequency attributes "b".

Frequencies are based on number of times mentioned in the presurvey. Brands and attributes used in the short version of the questionnaire are denoted with an asterisk.

\section{Laundry detergents}

Brands: Surf, Cold Power*, Almat, Bio*, Dynamo, Omo*, Fab, Spree*, Drive, Homebrand*

Attributes (actual wording):

- anti-bacterial $^{\mathrm{a}}$

- $\operatorname{modern}^{\mathrm{b}}$

- Australian-made ${ }^{\mathrm{b}, *}$

- bubbly $^{\mathrm{b}}$

- works well in cold water $^{\mathrm{a}}$

- $\quad$ light-blue colored ${ }^{\mathrm{b}, *}$
- gets out tough stains $^{\mathrm{a}, *}$

- gets rid of ground-in dirt ${ }^{\mathrm{a}}$

- gentle to clothes ${ }^{\mathrm{a}, *}$

- environmentally friendly ${ }^{\mathrm{a}}$

- $\quad$ gentle on $\operatorname{skin}^{\mathrm{a}, *}$

- $\quad$ low-priced ${ }^{\mathrm{a}, *}$

\section{Sports shoes}

Brands: New Balance, Asics*, Fila, K-Swiss, Dunlop*, Nike*, Adidas, Reebok*, Puma, Converse*

Attributes (actual wording):

- $\operatorname{cool}^{\mathrm{a}, *}$

- athletic-looking $^{\mathrm{a}}$

- springy, bouncy $^{\mathrm{b}}$

- American $^{\mathrm{b}, *}$

- Australian ${ }^{\mathrm{b}}$

- comfortable $^{\mathrm{a}_{*} *}$
- proper foot support $^{\mathrm{a}}$

- durable $^{\mathrm{a}, *}$

- attractive-looking $^{\mathrm{a}}$

- bright colors ${ }^{\mathrm{b}, *}$

- cheap image $^{\mathrm{a}, *}$

- shock-absorbing $^{\mathrm{a}}$ 
Table 2

Univariate effects of the methodological factors on the stability of brand-attribute associations (double positive associations as a percentage of all associations)

\section{Stability $(\%)$}

Methodological factor Low High $\quad \begin{array}{r}\text { Significance } \\ \text { at } p<.002\end{array}$

Favorite brand $($ low $=$ no, high $=$ yes $)$

49

71 sig.

Familiar brand (low familiarity, high

43

58

sig.

familiarity)

Task ease $($ low $=$ long, high $=$ short $)$

61

sig.

English comprehension (low = other

47

58

sig.

first language, high = English first

language)

Association direction (low $=$ attribute-

45

60

sig.

prompted, high $=$ brand-prompted)

Consumer involvement with the

product category (low involvement, high involvement)

Attribute importance (low importance,

56

52

n.s. high importance)

Note: The number of observations is 8,320 from 357 respondents. 
Table 3

Multivariate effects of the methodological factors on the stability of brand-attribute associations (binary: stable, unstable) via binary logistic regression
Methodological factor
B
Significance
S.E. Wald statistic at $p<.002$

\begin{tabular}{lcccc}
\hline Favorite brand & .743 & .108 & 47.74 & sig. \\
Brand familiarity & .215 & .042 & 25.99 & sig. \\
English comprehension & .342 & .083 & 16.94 & sig. \\
Consumer involvement & .005 & .001 & 15.23 & sig. \\
$\begin{array}{l}\text { Task ease } \\
\begin{array}{l}\text { Association-direction } \\
\text { (brand-prompted) }\end{array}\end{array}$ & .339 & .091 & 13.77 & sig. \\
$\begin{array}{l}\text { Attribute importance } \\
\text { Constant (intercept) }\end{array}$ & -.012 & .088 & 11.77 & sig. \\
\hline
\end{tabular}

Note: The number of observations is 8,320 from 357 respondents. 